\title{
ÜBER EINE ZUR FRATTINI-GRUPPE DUALE BILDUNG
}

\author{
NOBORU ITÔ
}

Es ist wohlbekannt, dass die Frattini-Gruppe einer endlichen Gruppe G, mit anderen Worten, der Durchschnitt aller maximalen Untergruppen von $G$, nilpotent ist. Offen ist dagegen bis jetzt die Frage, ob die Faktorgruppe $G / F^{*}(G)$ von $G$ nach dem Erzeugnis $F^{*}(G)$ aller minimalen Untergruppen eine besondere Struktur besitzt, z. B. ob sie auflösbar ist. Mehrere Schwierigkeiten sind der Grund hierfür: Einmal ist $G / F^{*}(G)$ im allgemeinen nicht nilpotent, zum anderen kennen wir die Struktur der einfachen und auflösbaren Gruppen (abgesehen von der Theorie von P. Hall) zu wenig. In der vorliegenden Note sollen teilweise Antworten auf die Frage nach der Auflösbarkeit von $G / F^{*}(G)$ gegeben werden.

\section{$\S 1$.}

Satz 1. Sei p eine ungerade Primzahl. Sei $G$ eine endliche Gruppe, deren sämtliche Untergruppen der Ordnung $p$ im Zentrum von $G$ liegen. Dann ist $G$ p-nilpotent, $d$. h. es gibt in $G$ einen Normalteiler $N$ derart, dass $G=N P$ und $N_{\frown} P=1$, wobei $P$ eine $p$-Sylowgruppe von $G$ ist.

Beweis. Offenbar erfüllt jede Untergruppe von $G$ die gleiche Bedingung wie $G$. Daher können wir annehmen, indem wir eine Induktion nach der Ordnung von $G$ durchführen, dass alle eigentlichen Untergruppen von $G p$-nilpotent sind. Ist nun $G$ nicht $p$-nilpotent, so liefert uns [1], Proposition 2 die folgende Faktorisierung von $G: G=P Q$, wo $P$ eine in $G$ invariante $p$-Sylowgruppe von $G$ ist, $Q$ eine in $G$ nicht invariante $p$-Sylowgruppe von $G$ ist $(q \neq p)$. Ferner ist $Q$ zyklisch. Sei $F(P)$ die Frattini-Gruppe von $P$. Ist $F(P)=1$, so ist $P$ abelsch vom Typ $(p, \ldots, p)$, woraus nach unserer Voraussetzung folgt, dass das Zentrum von $G$ die Gruppe $P$ umfasst. Dann ist $Q$ in $G$ invariant, was ein Widerspruch ist. Daher muss $F(P)$ von 1 verschieden sein. Nun betrachten wir die Faktorgruppe $G / F(P)$. Umfasst das Zentrum von $G / F(P)$ jede Unter-

Received May 1, 1955. 
gruppe der Ordnung $p$ von $G / F(P)$, so ist nach unserer Induktionsannahme $Q F(P) / F(P)$ invariant in $G / F(P)$, woraus die Invarianz von $Q$ in $G$ folgt, was ein Widerspruch ist. Daher enthält $G / F(P)$ mindestens eine Untergruppe $L / F(P)$ der Ordnung $p$, welche nicht im Zentrum von $G / F(P)$ liegt. Nach unserer Induktionsannahme ist die Gruppe $F(P) Q$ ein direktes Produkt $F(P) \times Q$. Nun betrachten wir den Zentralisator $K(F(P))$ von $F(P)$ in $G$. Dieser ist offenbar ein Normalteiler von $G$. Ist er von $G$ verschieden, so ist $Q$ in ihm invariant, woraus die Invarianz von $Q$ in $G$ folgt, was ein Widerspruch ist. Daher ist $K(F(P))=G$, mit anderen Worten, das Zentrum von $G$ umfasst $F(P)$. Insbesondere ist die Klasse von $P$ höchstens gleich zwei.

Für solche $p$-Gruppen gelten die Formeln: $(X Y)^{m}=X^{m} Y^{m}[X, Y]^{m(m-1) / 2}$ und $[X, Y]^{m}=\left[X^{m}, Y\right]$, wobei $X$ und $Y$ alle Elemente von $P$ durchlaufen und $m=0,1,2, \ldots$ (siehe etwa [3]). Wegen $p \neq 2$ folgt, dass diejenigen Elemente von $P$, deren Ordnung höchstens $p^{n}$ ist, eine Untergruppe $M(n, P)$ von $P$ bilden. Offenbar ist $M(n, P)$ invariant in $G$. Da $P$ nicht im Zentrum von $G$ liegt, ist $P \neq M(1, P)$. Ist $P \neq M(2, P)$, so betrachten wir das Produkt $M(2, P) Q$, welches nach unserer Induktionsannahme nilpotent ist. Wie oben dürfen wir annehmen, dass $M(2, P)$ im Zentrum von $G$ liegt. Nun betrachten wir die Faktorgruppe $G / M(1, P)$. Wegen $M(1, P / M(1, P))=M(2, P) / M(1, P)$ ist $G / M(1, P)$ nach unserer Induktionsannahme $p$-nilpotent. Daraus folgt die $p$-Nilpotenz von $G$, was ein Widerspruch ist. Daher gilt $P=M(2, P)$. Für jedes Paar $X, Y$ von Elementen von $P$ ist $[X, Y]^{p}=\left[X^{p}, Y\right]=1$. Mithin umfasst $M(1, P)$ die Kommutatorgruppe von $P$ und dann auch $F(P)$. Nun betrachten wir die Faktorgruppe $P / F(P)$ als Darstellungsmodul von $Q$. Dieser ist bekanntlich vollreduzibel (siehe etwa [2]). Ist er reduzibel, dann umfasst $P$ zwei verschiedene Normalteiler $P^{*}$ und $P^{* *}$ von $G$ mit $P / F(P)=P^{*} / F(P) \times P^{* *} / F(P)$ und $P^{*} \neq P \neq P^{* *}$. Wie oben dürfen wir annehmen, dass das Zentrum von $G$ sowohl $P^{*}$ als auch $P^{* *}$ umfasst, dann aber auch $P$, woraus die $p$-Nilpotenz von $G$ entgegen unserer Annahme folgt. Somit ist $P / F(P)$ irreduzibel bei $Q$. Insbesondere ist $F(P)=$ $M(1, P)$. Wir setzen $Q=\{z\}$ und $L=\{x\}$, wobei $L$ eine solche Untergruppe von $P$ der Ordnung $p^{2}$ sei, dass das Zentrum von $G / F(P)$ nicht $L F(P) / F(P)$ umfasst. Da $x^{p}$ im Zentrum von $G$ enthalten ist, so gilt $\left(z x z^{-1}\right)^{p}=z x^{p} z^{-1}=x^{p}$. Dann hat $[z, x]$ nach obiger Formel die Ordnung p, ist also ein Element von $F(P)$. Daher ist $L F(P)$ in $G$ invariant. Da $P / F(P)$ unter $Q$ irreduzibel ist, folgt $P=L F(P)$, woraus sich ergibt, dass $P$ zyklisch ist. Insbesondere ist $P=L . \quad$ Da $[z, x]$ ein 
Element des Zentrums von $G$ ist, ist $[z, x]^{q^{m}}=\left[z^{q^{m}}, x\right]=1$ für hinreichend grosse $m$ und $[z, x]^{p}=\left[z, x^{p}\right]=1$. Daraus ergibt sich $z x=x z$. Dann ist $G$ entgegen unserer Annahme $p$-nilpotent.

Bemerkung. Der entsprechende Satz für $p=2$ gilt nicht mehr, wie uns Herr Huppert mitgeteilt hat. Wir geben hier ein für unsere $Z$ wecke geeigneteres Beispiel an: Sei $q$ eine Primzahl grösser als $3, S L(2, q)$ die spezielle lineare Gruppe vom Grad 2 über dem Primkörper der Charakteristik q. Eine einfache Rechnung zeigt, dass das Zentrum von $S L(2, q)$ die einzige Untergruppe von $S L(2, q)$ der Ordnung 2 ist. Andererseits ist wohlbekannt, dass $S L(2, q)$ vollkommen ist, mit anderen Worten, die Kommutatorgruppe von $S L(2, q)$ ist $S L(2$, $q)$ selbst. Satz 1 mit $p=2$ gilt daher nicht für $\operatorname{SL}(2, q)$.

$\S 2$.

Sei $G$ eine Gruppe. $F(G)$ bezeichne die Frattini-Gruppe von $G, F^{*}(G)$ das Erzeugnis aller minimalen Untergruppen von $G$.

Hilfssatz. Sei $S$ eine Eigenschaft endlicher Gruppen, welche, wenn sie in $G$ gilt, auch für jede Untergruppe von $G$ gilt. $G$ sei eine S-Gruppe, d. h. eine Gruppe mit der Eigenschaft S. Sei $G / F^{*}(G)$ nicht aufösbar. Dann gibt es eine S-Gruppe $K$ derart, dass (i) $K / F(K)$ minimal einfach ist und (ii) $F(K)$ umfasst $F^{*}(K)$; dabei heisst eine Gruppe $X$ minimal einfach, wenn (a) $X$ einfach und nicht aufösbar ist und (b) jede eigentliche Untergruppe von $X$ auflösbar ist.

Bemerkung. Wir nennen $K$ eine $S$-Kerngruppe. Offen bleibt die Frage, ob es überhaupt für irgendein $S$ eine Kerngruppe wirklich gibt oder nicht.

Beweis. Sei $K$ eine Untergruppe von $G$ derart, dass für jede eigentliche Untergruppe $L$ von $K$ die Faktorgruppen $L / F^{*}(L)$ auflösbar sind, aber die Faktorgruppe $K / F^{*}(K)$ nicht auflösbar ist. Da $G / F^{*}(G)$ nicht auflösbar ist, gibt es natürlich solche $K$. Zunächst beweisen wir, dass $F(K)$ die Gruppe $F^{*}(K)$ umfasst. Dazu nehmen wir an, dass dies nicht gilt. Dann gibt es eine eigentliche Untergruppe $L$ von $K$ derart, dass $L F^{*}(K)=K$ ist. Offenbar umfasst $F^{*}(K) \frown L$ die Gruppe $F^{*}(L)$. Daraus folgt die Auflösbarkeit von $K / F^{*}(K)$, da nach unserer Annahme über $K$ die Faktorgruppe $L / F^{*}(L)$ auflösbar ist und $K / F^{*}(K)$ und $L / F^{*}(K) \frown L$ miteinander isomorph sind. Das ist aber ein Widerspruch. Daher liegt $F^{*}(K)$ in $F(K)$. 
Sei nun $M$ ein Normalteiler von $K$ derart, dass (a) $F(K)$ in $M$ liegt und (b) die Faktorgruppe $M / F(K)$ kein trivialer Normalteiler von $K / F(K)$ ist. Dann gibt es eine Untergruppe $H$ von $K$ mit $K=H M$. Die Faktorgruppe $K / M$ ist daher isomorph zu $H / H M$. Da $F^{*}(K)$ in $F(K)$ und dieses in $M$ liegt, umfasst $H M$ die Gruppe $F^{*}(H)$. Nach unserer Induktionsannahme ist $H / F^{*}(H)$ auflösbar, daher auch $H / H M$ und unserer $K / M$. Andererseits ist $M / F(K)$ auflösbar, da $M / F^{*}(M)$ nach Annahme aufösbar ist und $F^{*}(M)$ in $F^{*}(K)$, dieses aber in $F(K)$ liegt. Daher ist $K / F(K)$ aufösbar, folglich auch $K / F^{*}(K)$, was ein Widerspruch ist. Somit ist $K / F(K)$ einfach.

Satz 2. Sei S die folgende Eigenschaft:

Ist $p$ ein Primteiler der Ordnung von $G$, so gilt mit Ausnahme von höchstens zwei Primzahlen: Das Zentrum einer $p$-Sylowgruppe $P$ von $G$ enthält alle Elemente der Ordnung $p$ von G. Die Primzahl 2 sei stets Ausnahmeprimfaktor, falls 2 ein Teiler der Ordnung von $G$ ist.

Dann gibt es keine S-Kerngruppe.

Beweis. Sei $K$ eine S-Kerngruppe für diesen Fall. $p$ sei ein Primteiler der Ordnung von $K$, welcher kein Ausnahmeprimteiler ist. $P$ sei eine $p$-Sylowgruppe von $K$. Bekanntlich ist $F(K)$ nilpotent. Nach pem Hilfssatz liegt $F^{*}(K)$ in $F(K)$. Als Untergruppe von $F(K)$ ist $F^{*}(P)$ die einzige $p$-Sylowgruppe von $F^{*}(F(K))=F^{*}(K)$. Insbesondere ist $F^{*}(P)$ ein Normalteiler von $K$. Nun betrachten wir den Zentralisator von $F^{*}(P)$ in $K$, welcher nach unserer Voraussetznng $P$ umfasst. Da $K / F(K)$ einfach ist, ist entweder $P$ eine $p$-Sylowgruppe von $F(K)$ oder das Zentrum von $K$ umfasst $F^{*}(P)$. Der zweite Fall widerspricht jedoch Satz 1. Daher dürfen wir annehmen, dass der erste Fall eintritt. Wegen der Nilpotenz von $F(K)$ (siehe etwa [3]) ist dann $P$ ein Normalteiler von $K$. Nach einem bekannten Satz von Schur (siehe 3) umfasst $K$ dann eine Untergruppe $H$ mit $K=H P$. Eine maximale Untergruppe von $K$, welche $H$ umfasst, enthält demnach $P$ nicht. Das ist ein Widerspruch. Also gibt es keine Primzahl $p$ der angenommenen Art und die Ordnung von $K$ enthält höchstens zwei verschiedene Primfaktoren. Cann ist $K / F(K)$ nach einem wohlbekannten Satz von Burnside (siehe [2]) auflösbar, womit ein Widerspruch erreicht ist.

\section{LITERATUR}

[1] N. Ito: Note on ( $L M)$-groups of finite orders, Kōdai math. Sem. Reports 1951, S. 1-6. 
[2] A. Speiser: Die Theorie der Gruppen von endlicher Ordnung, 3. Aufl. 1937.

[3] H. Zassenhaus: Lehrbuch der Gruppentheorie, Bd. I. 1937.

Mathematisches Institut,

Universität zu Nagoya 